\section{But is the platter really silver?}

\section{To the Editor:}

The article by South et al. ${ }^{1}$ in the November 2009 issue of

Genetics in Medicine is an interesting report on de facto genomic screening of a population with colon cancer. As expected, immunohistochemical (IHC) stains for mismatch repair proteins were abnormal in a significant percentage $(21.1 \%)$ of the resected malignancies. However, both the authors and, one might safely venture, many readers were perplexed and perhaps dismayed by the rather anemic enthusiasm for genetic health care among these patients. Among those individuals who were considered excellent candidates for genetic counseling and potential testing for deleterious germline mutations in the mismatch repair genes, only one of four ( 9 of 54 or $26.5 \%$ ) made an appointment. This was world-class genomic medicine served on a silver platter, and most guests sent it back to the kitchen! Sacre Bleu! How poorly does this bode for the much-anticipated embrace of Personalized Medicine by our rank-and-file members of the health care consummerate? This is the easy stuff, well accepted, endorsed by the leading authorities and pundits, and reimbursed. Clinical utility even gives it a positive nod.

The easy solution might be to shrug shoulders and move on to the next project. However, one might consider this an opportunity to take yet another fresh look at how patients think and feel about genetic health care as it evolves into a more standard component of health care and how we as a health care profession are facilitating this evolution. South et al. ${ }^{1}$ reported that "Common barriers identified by the genetic counselors were cost, time, and lack of interest." We might benefit from further analysis of these impediments. In addition, $12 \%$ of patients with abnormal IHC results were prisoners, and 8.8\% were deceased "by the time genetics attempted to call out their abnormal IHC result." This latter observation suggests an arrangement where the clinical genetics team did not operate in synchrony with the primary care provider, the colorectal surgeon, or the oncologist, each of whom would be well-informed regarding the details of their patients' health, at least to the extent that they would not call results to patients who had passed away. To what extent had patients been made aware of the IHC testing, and to what extent had the surgeons of record and their teams been involved in the IHC screening efforts? If the "routine" nature of IHC screening were folded into all of the other routine preoperative information and the surgical candidates did not grasp the concept that an abnormal result would trigger a cascade of genetic analysis, counseling, follow-up, and expense, however minimal, these patients, still recovering from major surgery, might justifiably view the genetics team as intruders. As South et al. ${ }^{1}$ point out, the solution may well rest with the incorporation of genetic counseling within the scheduled follow-up appointments with surgery or oncology. The incorporation, although, might be most effective if it is considered phenomenological rather than merely an isolated event. In this regard, genetic health care is seen too often as a mere appendage to "regular" health care, with nongeneticists comfortable in the role of bemused but distant observers and less committed to folding genetic thinking and creativity into their own health care batter. We should have no doubt that South et al., ${ }^{1}$ based on their long experience in providing world-class genetic health care, pursued the goal of full genetic integration with the utmost of vigor. But their predicament serves as a warning to us all that effective genetic health care requires deep and broad commitment to developing strong partnerships with the full range of other health care providers and their patients. And it looks like it will be up to us, the genetics community, to take the first step in forging those relationships and creating systems which will make incorporation of genetics a routine part of health care whenever and wherever it has been shown to benefit patients. It is difficult to mourn the loss of an opportunity to get patients into the clinic for what we rightly see as fabulous, effective, genetically oriented health care without scrutinizing whether some aspect of the fundamental structure of our health care system may constitute the single most effective barrier to good genomic medicine.

Universal newborn screening has a number of similarities: "routine" population screening for genetic conditions using protein markers, follow-up systems with no prior relationship with the patient, information delivery outside of regularly scheduled visits with providers who may feel "out of the loop," trouble with appointments, and sick and inaccessible patients. It would not be unreasonable to suggest that programs of adult genetic screening might gain some insight from the experiences of state newborn screening programs, which have tested millions of infants annually for many decades.

Scott D. McLean, MD

Red Herring Genetics

San Antonio, Texas

Disclosure: The author declares no conflict of interest.

\section{REFERENCE}

1. South CD, Yearsley M, Martin E, Arnold M, Frankel W, Hampel H. Immunohistochemistry staining for the mismatch repair proteins in the clinical care of patients with colorectal cancer. Genet Med 2009;11:812-817. 